OPEN ACCESS

Edited by:

Manoj K. Sharma,

Jawaharlal Nehru University, India

Reviewed by:

Fumihiko Sato,

Kyoto University, Japan

Thu Thuy Dang,

University of British Columbia

Okanagan, Canada

*Correspondence:

Sairam Rudrabhatla

svr11@psu.edu

Specialty section: This article was submitted to

Plant Biotechnology,

a section of the journal

Frontiers in Plant Science

Received: 06 July 2020 Accepted: 09 November 2020

Published: 08 December 2020

Citation:

Deguchi M, Kane S, Potlakayala S,

George H, Proano R, Sheri V, Curtis WR and Rudrabhatla S (2020) Metabolic Engineering Strategies of Industrial Hemp (Cannabis sativa L.): A Brief Review of the Advances and

Front. Plant Sci. 11:580621. doi: 10.3389/fp/s.2020.580621

\section{Metabolic Engineering Strategies of Industrial Hemp (Cannabis sativa L.): A Brief Review of the Advances and Challenges}

\author{
Michihito Deguchi', Shriya Kane'2, Shobha Potlakayala', Hannah George ${ }^{1}$, Renata Proano', \\ Vijay Sheri', Wayne R. Curtis ${ }^{3}$ and Sairam Rudrabhatla ${ }^{1 *}$ \\ 'The Central Pennsylvania Research and Teaching Laboratory for Biofuels, Penn State Harrisburg, Middletown, PA, United \\ States, ${ }^{2}$ School of Medicine, Georgetown University, Washington, DC, United States, ${ }^{3}$ Department of Chemical Engineering, \\ The Pennsylvania State University, University Park, PA, United States
}

Industrial hemp (Cannabis sativa L.) is a diploid $(2 n=20)$, dioecious plant that is grown for fiber, seed, and oil. Recently, there has been a renewed interest in this crop because of its panoply of cannabinoids, terpenes, and other phenolic compounds. Specifically, hemp contains terpenophenolic compounds such as cannabidiol (CBD) and cannabigerol (CBG), which act on cannabinoid receptors and positively regulate various human metabolic, immunological, and physiological functions. CBD and CBG have an effect on the cytokine metabolism, which has led to the examination of cannabinoids on the treatment of viral diseases, including COVID-19. Based on genomic, transcriptomic, and metabolomic studies, several synthetic pathways of hemp secondary metabolite production have been elucidated. Nevertheless, there are few reports on hemp metabolic engineering despite obvious impact on scientific and industrial sectors. In this article, recent status and current perspectives on hemp metabolic engineering are reviewed. Three distinct approaches to expedite phytochemical yield are discussed. Special emphasis has been placed on transgenic and transient gene delivery systems, which are critical for successful metabolic engineering of hemp. The advent of new tools in synthetic biology, particularly the CRISPR/Cas systems, enables environment-friendly metabolic engineering to increase the production of desirable hemp phytochemicals while eliminating the psychoactive compounds, such as tetrahydrocannabinol (THC).

Keywords: cannabinoid, CRISPR/Cas, Cannabis sativa, metabolic engineering, RNA interference

\section{INTRODUCTION}

There is evidence of the historical use of industrial hemp (Cannabis sativa L.) in human civilization for both its phytochemical and lignocellulosic biomass properties. Hemp's native origin appears to be Eurasia with distribution around the world primarily as a fiber crop (Frassinetti et al., 2018). The emergence of petrochemical-derived polymer fiber sources decreased the demand for hemp; however, its use as a food and feed supplement has increased because it contains essential fatty acids (omega-6 and omega-3), easily digestible proteins (albumin and edestin) and enhanced levels of the amino acid arginine, which has indications for cardiovascular 
health (Bonini et al., 2018). Recently, more attention has been given to its rich repertoire of pharmaceutical compounds (Izzo et al., 2009; Degenhardt et al., 2017).

To date, more than 540 phytochemicals have been reported in hemp (Andre et al., 2016). Of these, cannabidiol (CBD) is generally most abundant and promising phytochemical as it has shown potential as a therapeutic agent in preclinical models of central nervous system diseases (Hill et al., 2012). Unlike tetrahydrocannabinol (THC), which has been associated with numerous side effects (Russo, 2011), CBD has an extremely safe profile in humans (Pertwee, 2008; Zuardi, 2008). Recently, the FDA has approved CBD (epidiolex) as an anticonvulsant drug (Brown and Winterstein, 2019). Additionally, hemp produces other cannabinoids and terpenes that exhibit a wide array of pharmacological properties (McPartland and Russo, 2001; Izzo et al., 2009; Russo, 2011). Since there is an increased demand for hemp-derived medicinal products, it is imperative to adapt biotechnological methodologies to generate new hemp strains with significant quantities of phytochemicals of medical interest.

Synthetic pathways for representative cannabinoids and terpenes have been elucidated (Figure 1), but the metabolic engineering of the pathway genes, enzymes, and metabolite regulation remains to be studied (Bonini et al., 2018). Thus, the development of an efficient regeneration and stable transformation system is essential. In this review, we present the strategies of target gene selection for hemp metabolic engineering. Challenges and opportunities to utilize transient and stable gene expression approaches are also discussed toward achieving a reliable metabolic engineering system in hemp.

\section{STRATEGIES OF TARGET GENE SELECTION FOR ENHANCING PHYTOCHEMICAL YIELD IN HEMP}

Manipulation of single that genes encode biosynthetic enzymes attempt to target genes that regulate the supply of precursors for the synthetic pathway that usually regulate flux into the pathway (targeting precursor-synthesizing gene), or rate-limiting step enzyme coding genes (targeting phytochemical pathway gene). Genetic engineering now permits more explicit manipulation of these metabolic fluxes (O'Connor, 2015), where we suggest that the native Cannabis host represents an ideal platform for this commercialization that is currently limited by the limitations of applicability of current biotechnological tools.

\section{Targeting Phytochemical Pathway Gene}

A focus on rate-limiting step is particularly effective for demonstrating function, as it permits confirming biochemistry in the absence of a background. This is exemplified in work of Sirikantaramas et al. (2004) who introduced the tetrahydrocannabinolic acid synthase (THCAS) gene in tobacco to synthesize THCA from cannabigerolic acid (CBGA), which was exogenously through roots. With this strategy, the composition of cannabinoids can be modified to more desirable such as high cannabidiolic acid (CBDA) or CBGA contents by overexpression of $C B D A$ synthase $(C B D A S)$ or aromatic prenyl transferase/PT4, respectively. Knockout of THCA by silencing THCA synthase (THCAS) will be expected, given the fact that other plant-derived prenyl-compounds are difficult

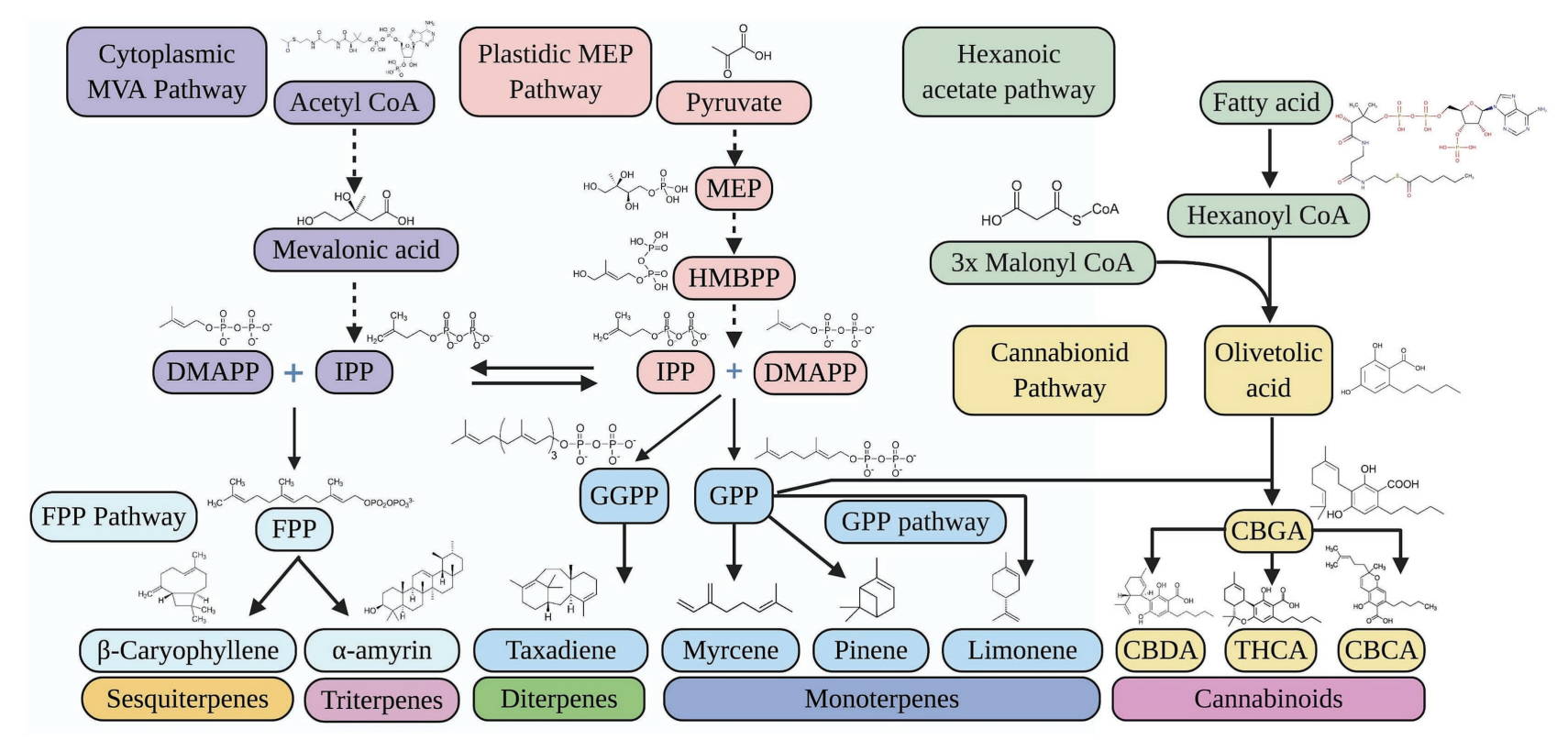

FIGURE 1 | Biosynthetic pathways for cannabinoids and terpenoids in hemp. CBCA, cannabichromenic acid; CBDA, cannabidiolic acid; CBGA, cannabigerolic acid; DMAPP, dimethylallyl diphosphate; FPP, farnesyl diphosphate; GGPP, geranylgeranyl pyrophosphate; GPP, geranyl diphosphate; HMBPP, (E)-4-hydroxy-3methyl-but-2-enyl pyrophosphate; IPP, isopentenyl diphosphate; MEP, methylerythritol phosphate; MVA, mevalonate; THCA, tetrahydrocannabinolic acid. Created with BioRender. 
to isolate from psychoactive THCA due to the structural similarity (Valliere et al., 2019). The quantity of CBGA will also be increased by simultaneous silencing of THCAS and CBDAS. Besides, silencing of both THCAS and CBDAS will expedite the production of minor cannabinoid, cannabichromenic acid (CBCA) which is difficult and expensive for pharmacological studies in clinical trials because of low abundance (Gülck and Møller, 2020).

\section{Targeting Precursor Synthesizing Gene}

Enzyme capacity for metabolism would be reasonably matched to the substrate availability for a pathway. Therefore, the ability to increase product formation with targeting phytochemical pathway gene will logically be quickly limited by the metabolite flux of upstream precursors (Balcke et al., 2017). In this scenario, metabolic engineering to increase precursor of synthetic pathway has been demonstrated to progressively increase downstream products ( $\mathrm{Ku}$ et al., 2020). For the engineering of cannabinoid synthesis genes, the supply of the high amount of geranyldiphosphate (GPP), which is a substrate to synthesize CBGA is essential. GPP is produced from isopentenyl diphosphate (IPP) in plastid (Figure 1), thus the overexpression of GPP synthase or activating MEP-pathway genes that resulted in higher IPP concentration in the plastid will be useful (Schachtsiek et al., 2018). Olivetolic acid, which is other substrate for CBGA synthesis, is formed from hexanoyl CoA by both tetraketide synthase and olivetolic acid cyclase. According to Stout et al. (2012), the concentration of hexanoyl-CoA paralleled the accumulation of the CBDA, which indicates that the synthesis of hexanoyl-CoA will be rate-limiting step in cannabinoid biosynthesis. Thus, overexpression of two isoforms for acylactivating enzyme (AAE) 1 and 3 will lead to increase the supply of hexanoyl-CoA. Importantly, comprehensive gene expression was studied in nine Cannabis strains with different phytochemical content, which revealed crosstalk between cannabinoid and terpene accumulation (Zager et al., 2019). Further study will provide an insight to understand which precursors need to be supplied in excess to increase the synthesis and accumulation of target metabolites.

\section{Pathway Activation}

Unlike microbial metabolite pathways that are often polycistronic, the genes for plant secondary metabolites are scattered throughout the vast plant genomes. The manipulation of genes that are linked though transcription factors provides a means to upregulate a pathway, but circumventing the signal transduction that precedes coordinated regulation such as a pathogen defense response. This strategy has been applied to many plant secondary metabolites, including anthocyanins in Arabidopsis (Liu et al., 2018; Outchkourov et al., 2018), flavonoids in tomato (Stracke et al., 2007; Luo et al., 2008), and alkaloids in Catharanthus roseus (Van Moerkercke et al., 2015; Pan et al., 2019) by upregulation of transcription factors. In Cannabis, van Bakel et al. (2011) identified several dozen transcription factors that are likely to play roles in the regulation of the THC synthesis pathway. Additionally, Marks et al. (2009) demonstrated the function of two MYB-domain transcription factor that seem to regulate the cannabinoid synthesis in the Cannabis trichome. These transcription factors are targets to activate cannabinoid synthesis. Notably, cannabinoid synthesis is expedited by UV light application or heavy metal (Zhang and Björn, 2009; Husain et al., 2019). The elucidation of signal transduction triggered by these elicitors may lead to the discovery of positive and negative regulators of signal transduction, which will be the target genes for hemp metabolic engineering.

\section{Alternative Platforms for Hemp Phytochemical Production}

The nature of metabolic engineering introduces the opportunity to not only examine a native production platform for a biochemical, but also the potential to move that biosynthesis into an alternative host. Microbial platforms have served as elegant platforms for the elucidation of plant metabolite function (Pyne et al., 2019), and will likely be extremely valuable in elucidating yet unknown enzymatic conversions in hemp cannabinoids. For higher value, and immediate market-driven production, platforms such as this can be expected to provide specific metabolites in hemp. Recently, CBDA was synthesized in yeast via the introduction of the MEP pathway, GPP pathway, hexanoic acetate pathway, and CBDA synthesis pathway (Zirpel et al., 2017; Luo et al., 2019). On the other hand, heterologous production in other plant species is still a challenge. It is not unusual for heterologous metabolite production platforms to lack physiological requirements for the high productivity that can be observed in native systems (Schachtsiek et al., 2018). In cannabinoid synthesis, toxicity effects must be considered, as several cannabinoid pathway metabolites such as CBGA and THCA cause cell death via apoptosis in host plant (Sirikantaramas et al., 2005). In hemp, olivetolic acid synthesized in cytosol is transferred to plastid, where olivetolic acid and geranyl-PP are converted into CBGA, which is finally released to apoplast (Gülck and Møller, 2020). It will be critical to elucidate the mechanism underlying transport and accumulation of metabolites and apply it to better hemp phytochemical production in other plant species commercially (Table 1). Looking toward the future of hemp "designer lines" to produce various phytochemicals, we now focus the remainder of this review on the challenge of advancing biotechnological methods of plant transformation and regeneration as it applies to hemp.

\section{Transgenic Gene Delivery Systems} Tissue Culture and Stable Transformation

To establish an efficient transformation system, the development of a hemp regeneration protocol is critical. Until recently, a variety of explants such as leaf, hypocotyl, cotyledon, stem, axillary bud, petioles, and shoot tips were tested with the combination of different auxins and cytokinins for the purpose of direct or indirect regeneration (Table 2). Lata et al. (2009a) demonstrated the induction of high-frequency shoot regeneration from nodal segments containing axillary buds using thidiazuron (TDZ). Lata et al. (2010) obtained the highest shoot induction rate at $0.5 \mu \mathrm{M}$ TDZ in callus, whereas Chaohua et al. (2016) 
TABLE 1 | Companies who have utilized genetic modification techniques to produce cannabinoids on an industrial scale.

Company and location
Canopy Growth Corp.; Smiths Falls, Canada
Ebbu;
Evergreen, CO, United States
Zenabis;
Vancouver, Canada
Farmako;
Frankfurt, Germany
Ginkgo Bioworks;
Boston, MA, United States
Croncos Group;
Toronto, Canada
Librede;
Carlsbad, CA, United States
Demetrix;
Emeryville, CA, United States
Maku Technologies; Durham, North Carolina, United States
InMed Pharamaceuticals; Vancouver, Canada

Renew Biopharma;

San Diego, CA, United States

Teewinot Life Sciences; Tampa, Florida, United States

Trait Biosciences Toronto, Canada

InPlanta Biotechnology; Lethbridge, Canada

Dewey Scientific;

Pullman, WA, United States

\section{Product detail}

Largest legal Cannabis company in the world and has partnerships/acquired the following companies: Ebbu; Spectrum Therapeutics; Canopy Innovation Lab; Storz \& Bickel; Ebba, Battelle, Apollo, and Scientus.

Developed CRISPR-Cas9 to produce plants that secrete only CBD and only CBG.

Sells both recreational and medical Cannabis and have the following subset companies: Vida, Zen Craft Grow, Namaste, Blazery, and Re-Up.

Turned to Zymomonas mobilis bacterium to make 180 cannabinoids, including THC and CBD to use in Cannabis-based drug therapies.

Synthetic-biology company that worked with the Croncos Group to manufacture pure CBD and other cannabinoids in yeast.

Focuses on advancing Cannabis research, technology, and product development. Has a brand portfolio that includes PEACE NATURALS, COVE, SPINACH, Lord Jones and PEACE+.

Synthetic-biology company with patent to use yeast (Saccharomyces cerevisiae) to synthesizing cannabinoids from sugars while being sustainable.

Uses Saccharomyces cerevisiae controlled fermentation to produce rare cannabinoids.

Focuses on producing rare, natural cannabinoids in yeast to increase research on cannabinoids. Produces enzymes with Escherichia coli biofermentation to yield cannabinoids through the process of biotransformation and other purification stages. The cannabinoids can also be converted to other rare cannabinoids.

Uses Chlamydomonas reinhardtii to produce cannabinoids and uses the cannabinoids to target certain receptors that contribute to brain inflammation and chronic pain. Has a patent for the NphB enzyme in cannabinoid synthesis.

Has a patent for a bioreactor designed to grow cannabinoid-producing microorganisms called CannSynthesis. Can produce 25 minor cannabinoids and are developing a library of cannabinoid analogs.

Identified a gene that when expressed in Cannabis leads to increased trichome production and upregulation of cannabinoids. Creates water-soluble cannabinoids, customizes cannabinoid profiles, and produces THC-free hemp.

Focuses on growing Cannabis with specific CBD/THC/terpenoid contents and breeding high CBD hemp.

Offers scientific insights to Cannabis producers to increase efficiencies and crop yields while decreasing crop inputs by looking at molecular biology and traditional breeding. demonstrated the highest shoot induction at $2.0 \mu \mathrm{M}$ TDZ in the cotyledon. Wielgus et al. (2008) tested various combinations of plant growth regulators and obtained regenerated plants on MS medium containing benzoic acid herbicide: DICAMBA. A cytokinin meta-topolin was also effective for shoot regeneration from nodal explants (Lata et al., 2016a). In vitro propagation has also been studied in C. sativa and reviewed by Lata et al. (2017). Recently, Kodym and Leeb (2019) established a photoautotropic micropropagation system and obtained a $97.5 \%$ rooting rate from the in vitro generated shoot tip cuttings.

Subsequently, a few successful Cannabis transformation systems were reported by Lata et al. (2017). Suspension cell culture and hairy roots were transformed via Agrobacterium, whereas the transformed tissues were not regenerated (Feeney and Punja, 2003). Hypocotyl was inoculated with Agrobacterium, and the shoot was produced in MS media containing 6-benzylaminopurine and zeatin, which resulted in complete hemp transgenic plant (Sirkowski, 2012, United States Patent application 20120311744A1). Nevertheless, Cannabis regeneration and stable transformation are still limited to specific varieties, and a reliable transformation protocol has not been established (Salentijn et al., 2019), partially because of limited breeding that could establish homogenous lines.
It is worth highlighting that plant mechanism underlying somatic embryogenesis (SE) and subsequent regeneration system has been elucidated, and key regulators of plant cell totipotency were identified (Figure 2; Horstman et al., 2017; MéndezHernández et al., 2019). Upregulation of morphologic regulator (MR) genes has promoted regeneration rate in both monocotyledonous species (Lowe et al., 2016; Mookkan et al., 2017; Hoerster et al., 2020) and dicotyledonous species (Deng et al., 2009; El Ouakfaoui et al., 2010; Florez et al., 2015). The alteration of gene expression on hemp MR would open the door to enhance $\mathrm{SE}$ and facilitate the acquisition of regenerated and transformed hemp plants and developing a synthetic seed technology for commercialization of hemp clones.

\section{Genome-Editing Technologies}

The CRISPR-Cas system currently emerged as a genome editing tool with the simplicity of target design, high efficiency of editing, multiplex knock-in/out ability, and low cost (Jaganathan et al., 2018). In plant metabolic engineering, CRISPR/Cas-mediated engineering is robust and convenient to generate knockouts of target genes via the DNA repair pathway: nonhomologous end-joining (NHEJ). Because of its ability to perform simultaneous gene knockouts, this 
TABLE 2 | Overview of previously reported tissue culture and stable transformation work in Cannabis sativa.

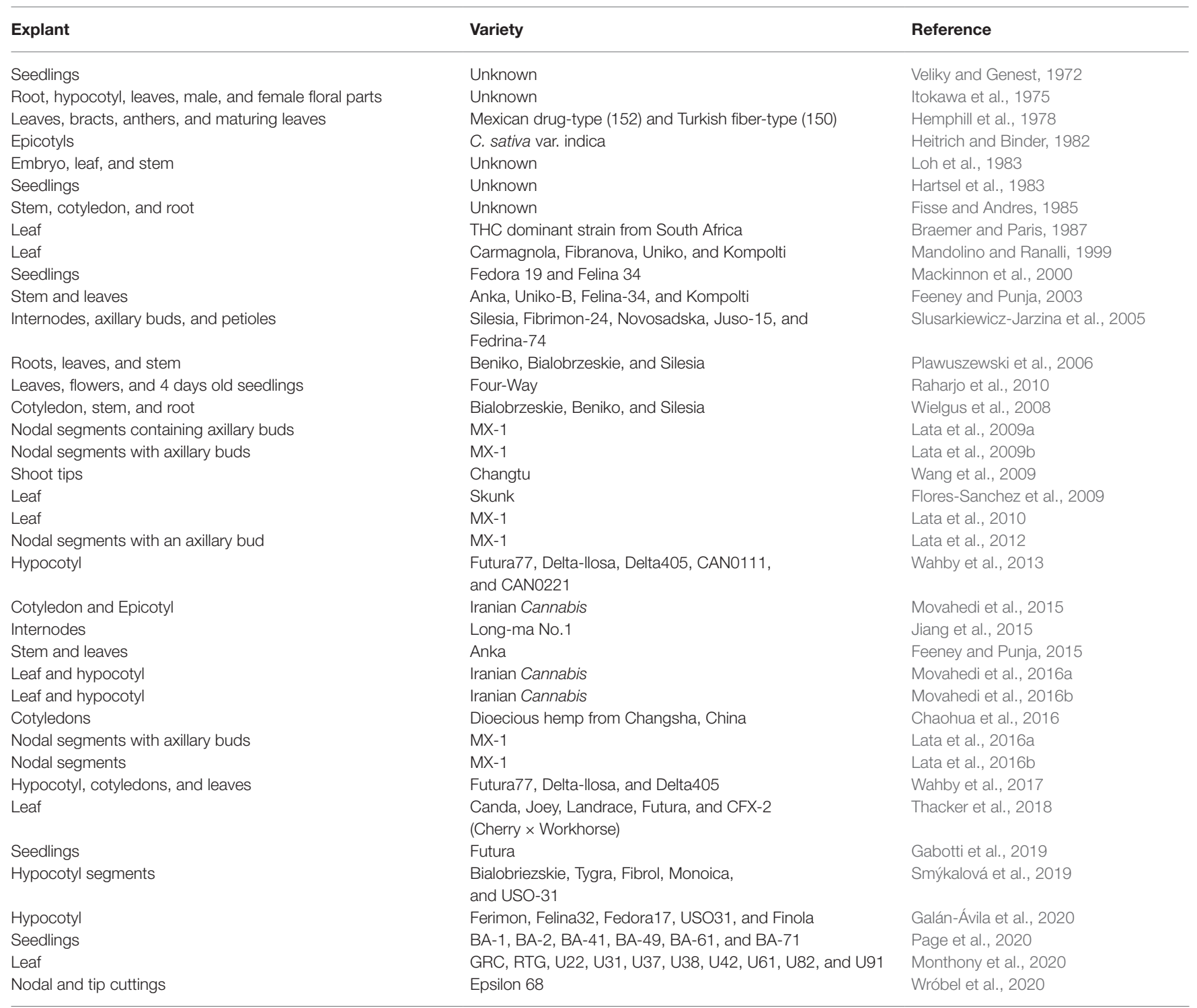

system can be used to eliminate undesired multiple branching pathways (Alagoz et al., 2016; Sun et al., 2017). Recent advances in multiplex genome-editing tools have made it possible to delete six tomato genes by expressing 12 gRNAs via the expression of a single CRISPR vector (Čermák and Curtin, 2017). This multiplex mutation system will be highly useful for hemp metabolic engineering too, for instance, simultaneous knocking down of THCAS and CBDAS to increase the synthesis of minor cannabinoids with potential pharmaceutical values that are present in hemp but have not been studied because of their low quantity.

Although many CRISPR tools are most effective for the knockout of endogenous genes, the overexpression of endogenous genes via homology-directed repair (HDR) is still a challenge in many plant species (Hahn et al., 2018). Targeting a cis-regulatory element is thus a viable alternative. A cis-regulatory element is a noncoding DNA region that contains binding sites for transcription factors or other proteins that control transcription. Recent research has demonstrated the enormous potential of editing cis-regulatory elements to regulate optimal gene expression denominated as "fine-tuning" (Shrestha et al., 2018; Wolter et al., 2019). Moreover, editing untranslated region (UTR) is also a useful approach for fine-tuning genes of interests given the fact that UTRs play an important role in the regulation of protein synthesis (Si et al., 2020).

Base editing has emerged as a newly developed technique that enables direct, irreversible conversion of one base pair to another without disruption of a gene or requiring a donor template (Mishra et al., 2020). Base editors such as cytosine base editor and adenine base editor are basically composed of cytosine or adenosine deaminase domain, respectively, and catalytically inactive CRISPR-Cas9 domain (Kang et al., 2018). The base-editing system can generate a single-base change or single nucleotide polymorphisms (SNP), thereby facilitating 
A

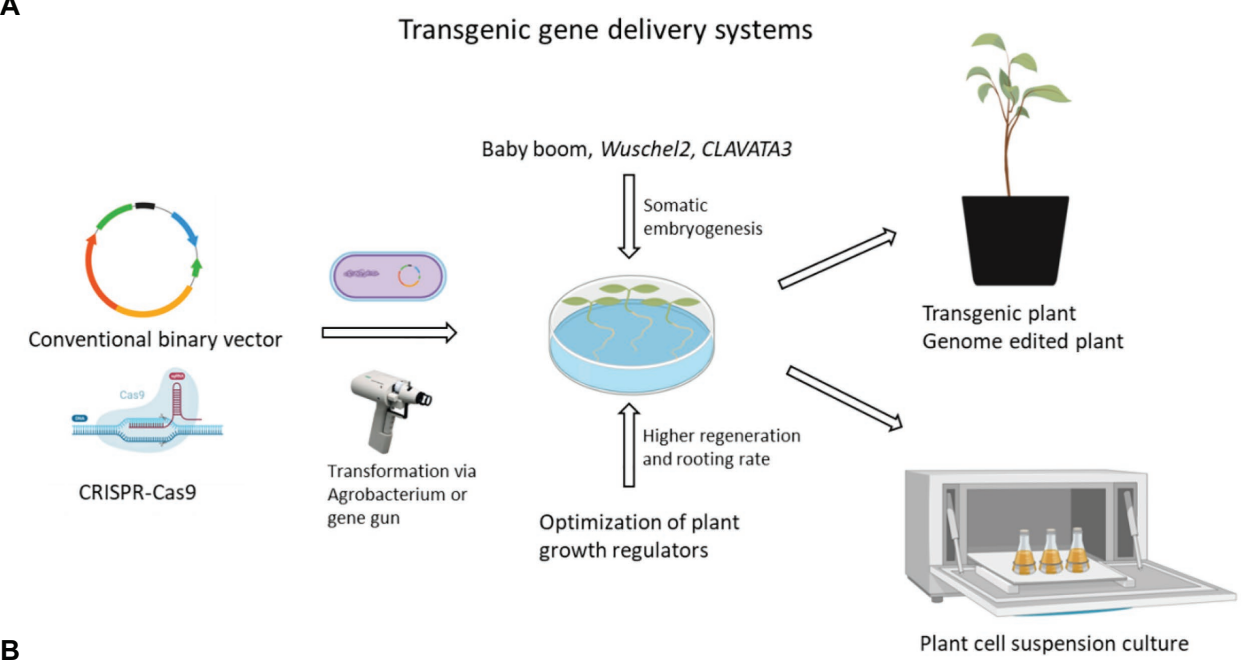

Non transgenic gene delivery systems

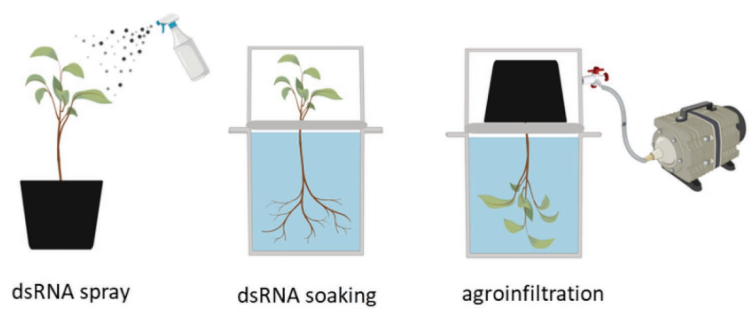

FIGURE 2 | A schematic strategy of transgenic and transient gene delivery system. These gene delivery systems aim to alter gene expression in hemp female flower, where cannabinoids and terpenes are preferentially synthesized and stored. (A) Transgenic gene delivery systems. Specialized metabolites and their precursor synthesis genes, transcription factor genes, and other hemp genes related to the supply of energy and reducing power might be engineered by introducing conventional binary vectors or CRISPR/Cas vectors. Hemp SE might be activated by the overexpression of positive regulator genes such as BBM and WUS2 or the downregulation of negative regulator genes such as CLAVATA3. (B) Three transient gene delivery systems. To overcome the instability of dsRNA, clay nanoparticles, liposomes, viruses, or bacteria might be used for the effective delivery of dsRNA. For successful agroinfiltration, vacuum infiltration is likely to be more efficient than syringe infiltration based on our preliminary experiments. Created with BioRender. CRISPR/Cas-mediated genome editing is likely to be most powerful method for hemp metabolic engineering.

plant breeding and basic research (Monsur et al., 2020). In Cannabis, more than 14,000 SNPs were genotyped in both drug type and fiber type strains (Sawler et al., 2015). Among them, several SNPs that are associated with Cannabinoid contents have been identified (Rotherham and Harbison, 2011; Onofri et al., 2015; Borna et al., 2017). These SNPs can be good targets for hemp metabolic engineering via base editing with the aim of altering the property of cannabinoid content.

It is important to note that these three editing methods: CRISPR/Cas mediated gene mutation via NHEJ, fine-tuning of gene expression via cis-regulatory elements, and base editing follow a cisgenic approach (Holme et al., 2013; Hou et al., 2014) and therefore, do not introduce any exogenous genes. Consequently, it is easier to obtain public acceptance for commercializing hemp products obtained via these methods, especially for hemp consumers that are usually averse to products obtained from genetically modified plants (Schluttenhofer and Yuan, 2017).

Of the three methods discussed, the CRISPR/Cas9 system shows the broadest utility, and it would be very beneficial to introduce CRISPR/Cas to hemp metabolic engineering for the following four reasons:

1. Hemp is a diverse and polymorphic species (Weiblen et al., 2015), and due to genome duplication, the gene copy number is high on many of hemp genes including phytochemical synthesis genes (van Bakel et al., 2011), which require more studies to identify functional genes. This makes genetic engineering more complex in this plant. On the contrary, CRISPR/Cas9 enables the knockout of several homologous genes via a single editing step (Jacobs et al., 2017).

2. Most hemp varieties do not self-pollinate; it is not feasible to obtain homozygous plants by self-pollination. However, CRISPR makes it possible to mutate or modify the gene of interest in both alleles at one editing step such that homozygous plants can be obtained in T0 editing generation. Shen et al. (2017) demonstrated that high efficiencies of site-specific double-stranded breaks 
allowed the isolation of mutants carrying homozygous mutated alleles of eight targeted genes simultaneously in rice.

3. In hemp, there is no established protocol for mutagenesis (Salentijn et al., 2019). Bielecka et al. (2014) showed that isolation of mutants from chemical mutagenesis screen is possible, but extremely difficult due to the anemophilous and dioecious nature of hemp. Genome editing technology allows for very specific gene editing and makes it easy to evaluate the effect of off-target gene modification. Therefore, this technology provides precision that is not possible with mutation breeding such as EMC mediated mutation that resulted in hundreds of unexpected mutations (Henry et al., 2014). Furthermore, a reverse genetic approach using the CRISPR/Cas mediated mutants will drastically accelerate the study of the function of synthetic pathway genes of specialized metabolites as there is gene silencing tools such as VIGS in hemp are just started to be developed by Schachtsiek et al. (2019).

4. Lastly, CRISPR/Cas system will drastically shorten the hemp breeding time. The methods commonly used in hemp breeding are "mass selection," "cross-breeding," "inbreeding," and "hybrid breeding" (Clarke and Merlin, 2016), and creating unique Cannabis strains via these traditional methods is time-consuming and takes both patience and persistence. Indeed, conventional Cannabis breeding has expanded to include the diverse composition of elite varieties, ranging from plants with no THCA to those with high concentrations of CBDA or terpenes (Russo, 2019). However, CRISPR/Cas-based editing system is able to carry out pyramiding multiple desirable traits such as phytochemical properties, degree of monoecy, length of vegetative cycle, and resistance to diseases and pest (Salentijn et al., 2019) in one editing step, which is not feasible or takes at least 10 years in conventional breeding (Schluttenhofer and Yuan, 2017).

\section{Transient Gene Delivery Systems Transient Gene Expression Via Agroinfiltration}

Agroinfiltration is a prominent methodology for temporarily expressing a gene of interest easily and rapidly. This technology was first explored in molecular studies, including transient reporter gene expression, promoter analysis, and proteinprotein interactions (Norkunas et al., 2018). Optimization of agroinfiltration has become a technique to produce vaccines, enzymes for industrial use, and secondary metabolites (Lai and Chen, 2012; Chen and Lai, 2013; Gleba et al., 2014; Reed and Osbourn, 2018). Agroinfiltration thereby provides an alternative method for stable transformation (Andrews and Curtis, 2005; Chen and Lai, 2015). Nevertheless, strong transient gene expression achieved via agroinfiltration is limited to some model crops, and agroinfiltration protocols for many agronomically important crops have only recently been optimized (King et al., 2015). Most recently, Deguchi et al. (2020) optimized the proper concentration of surfactant and antioxidants for Agrobacterium vacuum infiltration and achieved gene overexpression and silencing in hemp trichome. Schachtsiek et al. (2019) inoculated Agrobacterium carrying VIGS-vectors to Cannabis mature leaf which led to the reduction of $70 \%$ of gene expression in phytoene desaturase and magnesium chelatase subunit I. Enhanced efficiency of transient expression will not only pave the way for metabolic engineering but will also contribute to successful Agrobacterium inoculation into explants for stable transformation.

\section{Topical Application of dsRNA}

RNA interference (RNAi) is a gene regulation mechanism that induces the silencing of gene expression at the transcriptional or posttranscriptional level in eukaryotes (Petrick et al., 2013). In plants, this silencing mechanism has been used to confer resistance against pests and diseases by genetic transformation (Koch and Kogel, 2014). More recently, the topical application of dsRNA has emerged as an alternative to the generation of genetically modified plants. A prerequisite for the success of this technology is the efficient delivery of dsRNA to the plant. There have been several methods developed for achieving this, but the two most useful approaches are the soaking of the plant root and spray application on the surface of the plant (Andrade and Hunter, 2016). The topical application of dsRNA can be designed and tested much faster than the stable transformation of plants, which makes these approaches suitable for recalcitrant plants to stable transformation like hemp. Furthermore, double-stranded RNAs are present naturally in plants and normally degraded within a few days, rendering this technology more environment-friendly than others (Wang et al., 2011). Therefore, it is apt for hemp consumers that demand organic products and environmental sustainability of the crop. To date, this transient gene silencing approach has been successfully applied to control insects, fungi, and viruses (Koch et al., 2016; Mamta and Rajam, 2017; Dalakouras et al., 2019).

Attempts to convert the topical application of dsRNA to phytochemical production represent a new challenge. There are some reports from private companies regarding the development of RNAi spray and soaking protocols for Cannabis metabolic engineering, but the details of this technology have not been disclosed. The challenging task of identifying important targets in secondary metabolite pathways has been overcome with bioinformatic information such as hemp genomic and transcriptomic sequences, and a global map of metabolic pathways provided by the Kyoto Encyclopedia of Genes and Genomes (KEGG; Kanehisa and Goto, 2000). The instability of the naked dsRNA applied on plants is another barrier, but this could be rectified by using clay nanoparticles, liposomes, viruses, or bacteria as potential dsRNA carriers for spray application to achieve a longer-term gene-silencing effect (Joga et al., 2016; Mitter et al., 2017). Even if dsRNA technology has limited field applicability due to economic considerations, it could provide an exceptional tool for plant improvement in experimental settings. 


\section{DISCUSSION}

To date, production of improved hemp through the genetic engineering has been limited due to low shooting and rooting efficiency. However, newly emerging biotechniques are anticipated to overcome this barrier. The most powerful approach is likely to involve manipulation of MR to enhance the developmental pathway of somatic embryogenesis. Introduction of genes into the hemp genome does not appear to be limiting as for example the creation of "hairy roots;" however, the transition of that cell into a viable plant requires a major reprogramming of plant cell development. Using appropriate surfactant and antioxidant reagents, Agrobacterium infection to hemp explant became no longer a difficult step via vacuum infiltration (Deguchi et al., 2020). Once somatic embryogenesis is achieved by altering the transient expression of morphologic regulator genes, hemp explants would regenerate at a high rate and be available for further genetic modifications with value added traits. If highly efficient somatic cell conversion can be achieved, this technological advance can even be applied to rapid propagation of enhanced hemp phenotypes with some technologies now advancing to synthetic seeds. Interestingly, Maher et al. (2020) demonstrated that MRs worked compatibly with the CRISPR/Cas system in various dicot species.

Recently, hemp phytochemical pathway genes have been extensively studied via omics approaches (Braich et al., 2019; Vincent et al., 2019; Gao et al., 2020). However, the

\section{REFERENCES}

Alagoz, Y., Gurkok, T., Zhang, B., and Unver, T. (2016). Manipulating the biosynthesis of bioactive compound alkaloids for next-generation metabolic engineering in opium poppy using CRISPR-Cas 9 genome editing technology. Sci. Rep. 6:30910. doi: 10.1038/srep30910

Andrade, E. C., and Hunter, W. B. (2016). "RNA interference - natural genebased technology for highly specific pest control (HiSPeC)" in RNA interference. ed. I. Y. Abdurakhmonov (IntechOpen).

Andre, C. M., Hausman, J. F., and Guerriero, G. (2016). Cannabis sativa: the plant of the thousand and one molecules. Front. Plant Sci. 7:19. doi: 10.3389/ fpls.2016.00019

Andrews, L. B., and Curtis, W. R. (2005). Comparison of transient protein expression in tobacco leaves and plant suspension culture. Biotechnol. Prog. 21, 946-952. doi: 10.1021/bp049569k

Balcke, G. U., Bennewitz, S., Bergau, N., Athmer, B., Henning, A., Majovsky, P., et al. (2017). Multi-Omics of tomato glandular trichomes reveals distinct features of central carbon metabolism supporting high productivity of specialized metabolites. Plant Cell 29, 960-983. doi: 10.1105/tpc.17.00060

Bielecka, M., Kaminski, F., Adams, I., Poulson, P., Sloan, R., Li, Y., et al. (2014). Targeted mutation of $\Delta 12$ and $\Delta 15$ desaturase genes in hemp produce major alterations in seed fatty acid composition including a high oleic hemp oil. Plant Biotechnol. 12, 613-623. doi: 10.1111/pbi.12167

Bonini, S. A., Premoli, M., Tambaro, S., Kumar, A., Maccarinelli, G., Memo, M., et al. (2018). Cannabis sativa: a comprehensive ethnopharmacological review of a medicinal plant with a long history. J. Ethnopharmacol. 227, 300-315. doi: 10.1016/j.jep.2018.09.004

Borna, T., Salami, S. A., and Shokrpour, M. (2017). High resolution melting curve analysis revealed SNPs in major cannabinoid genes associated with drug and non-drug types of cannabis. Biotechnol. Biotechnol. Equip. 31, 839-845. doi: 10.1080/13102818.2017.1333456

Braemer, R., and Paris, M. (1987). Biotransformation of cannabinoids by a cell suspension culture of Cannabis sativa L. Plant Cell Rep. 6, 150-152. doi: $10.1007 / \mathrm{BF} 00276675$ characterization of function on most of phytochemical pathway genes remains to be studied. Advancement of molecular biology tools and the establishment of a hemp transformation system will not only achieve a diverse of hemp new varieties with improved quality and/or quantity of phytochemicals but also further intensify the investigation of other minor cannabinoid and terpene synthesis genes as well as several representative cannabinoid synthesis genes to expand the pharmacological potential of the hemp biochemical production platform.

\section{AUTHOR CONTRIBUTIONS}

SR and MD designed the concept for the review paper. MD, SK, and SP organized and wrote the manuscript. SR, WC, VS, $\mathrm{RP}$, and HG edited the manuscript. All authors contributed to the article and approved the submitted version.

\section{ACKNOWLEDGMENTS}

We would like to acknowledge support from the PA Options for Wellness and Penn State Harrisburg School of Science, Engineering, and Technology; Dr. Zhanyuan Zhang, Ms. Fatima Khan, and Ms. Karen Charleston for help with the manuscript. We thank the American Journal Experts (AJE) for English language editing and BioRender for their image creator.

Braich, S., Baillie, R. C., Jewell, L. S., Spangenberg, G. C., and Cogan, N. O. I. (2019). Generation of a comprehensive transcriptome atlas and transcriptome dynamics in medicinal Cannabis. Sci. Rep. 9:16583. doi: 10.1038/ s41598-019-53023-6

Brown, J. D., and Winterstein, A. G. (2019). Potential adverse drug events and drug-drug interactions with medical and consumer cannabidiol (CBD) use. J. Clin. Med. 2019:989. doi: 10.3390/jcm8070989

Čermák, T., and Curtin, S. J. (2017). Design and assembly of CRISPR/Cas9 reagents for gene knockout, targeted insertion, and replacement in wheat. Methods Mol. Biol. 1679, 187-212. doi: 10.1007/978-1-4939-7337-8_12

Chaohua, C., Gonggu, Z., Lining, Z., Chunsheng, G., Qing, T., Jianhua, C., et al. (2016). A rapid shoot regeneration protocol from the cotyledons of hemp (Cannabis sativa L.). Ind. Crop. Prod. 83, 61-65. doi: 10.1016/j. indcrop.2015.12.035

Chen, Q., and Lai, H. (2013). Agroinfiltration as an effective and scalable strategy of gene delivery for production of pharmaceutical proteins. Adv. Tech. Biol. Med. 1:103. doi: 10.4172/2379-1764.1000103

Chen, Q., and Lai, H. (2015). Gene delivery into plant cells for recombinant protein production. Biomed. Res. Int. 2015:932161. doi: 10.1155/2015/ 932161

Clarke, R. C., and Merlin, M. D. (2016). Cannabis domestication, breeding history, present-day genetic diversity, and future prospects. Crit. Rev. Plant Sci. 35, 293-327. doi: 10.1080/07352689.2016.1267498

Dalakouras, A., Wassenegger, M., Dadami, E., Ganopoulos, I., Pappas, M. L., and Papadopoulou, K. (2019). GMO modified organism-free RNAi: exogenous application of RNA molecules in plants. Plant Physiol. 182, 38-50. doi: 10.1104/pp.19.00570

Degenhardt, F., Stehle, F., and Kayser, O. (2017). “The biosynthesis of cannabinoids" in Handbook of cannabis and related pathologies: Biology, pharmacology, diagnosis, and treatment. ed. V. R. Preedy (London: Academic Press), 13-23.

Deguchi, M., Bogush, D., Weeden, H., Spuhler, Z., Potlakayala, S., Kondo, T., et al. (2020). Establishment and optimization of a hemp (Cannabis sativa L.) agroinfiltration system for gene expression and silencing studies. Sci. Rep. 10:3504. doi: 10.1038/s41598-020-60323-9 
Deng, W., Luo, K., Li, Z., and Yang, Y. (2009). A novel method for induction of plant regeneration via somatic embryogenesis. Plant Sci. 177, 43-48. doi: 10.1016/j.plantsci.2009.03.009

El Ouakfaoui, S., Schnell, J., Abdeen, A., Colville, A., Labbe, H., Hand, S., et al. (2010). Control of somatic embryogenesis and embryo development by AP2 transcription factors. Plant Mol. Biol. 74, 313-326. doi: 10.1007/ s11103-010-9674-8

Feeney, M., and Punja, Z. K. (2003). Tissue culture and agrobacterium-mediated transformation of hemp (Cannabis sativa L.). In Vitro Cell Dev. Biol. Plant 39, 578-585. doi: 10.1079/ivp2003454

Feeney, M., and Punja, Z. K. (2015). Hemp (Cannabis sativa L.). Methods Mol. Biol. 1224, 319-329. doi: 10.1007/978-1-4939-1658-0_25

Fisse, J., and Andres, J. (1985). Organogenesis and biosynthesis in an in vitro culture of Cannabis sativa L. Boletin da Acad. Galega de Cienc. 4, 57-67.

Flores-Sanchez, I. J., Pec, J., Fei, J., Choi, Y. H., Dusek, J., and Verpoorte, R. (2009). Elicitation studies in cell suspension cultures of Cannabis sativa L. J. Biotechnol. 143, 157-168. doi: 10.1016/j.jbiotec.2009.05.006

Florez, S. L., Erwin, R. L., Maximova, S. N., Guiltinan, M. J., and Curtis, W. R. (2015). Enhanced somatic embryogenesis in Theobroma cacao using the homologous BABY BOOM transcription factor. BMC Plant Biol. 15:121. doi: 10.1186/s12870-015-0479-4

Frassinetti, S., Moccia, E., Caltavuturo, L., Gabriele, M., Longo, V., Bellani, L., et al. (2018). Nutraceutical potential of hemp (Cannabis sativa L.) seeds and sprouts. Food Chem. 262, 56-66. doi: 10.1016/j.foodchem.2018.04.078

Gabotti, D., Locatelli, F., Cusano, E., Baldoni, E., Genga, A., Pucci, L., et al. (2019). Cell suspensions of Cannabis sativa (var. Futura): effect of elicitation on metabolite content and antioxidant activity. Molecules 24:4056. doi: 10.3390/ molecules 24224056

Galán-Ávila, A., García-Fortea, E., Prohens, J., and Herraiz, F. J. (2020). Development of a direct in vitro plant regeneration protocol from Cannabis sativa L. seedling explants: developmental morphology of shoot regeneration and ploidy level of regenerated plants. Front. Plant Sci. 11:645. doi: 10.3389/ fpls.2020.00645

Gao, S., Wang, B., Xie, S., Xu, X., Zhang, J., Pei, L., et al. (2020). A highquality reference genome of wild Cannabis sativa. Hortic. Res. 7:73. doi: 10.1038/s41438-020-0295-3

Gleba, Y. Y., Tusé, D., and Giritch, A. (2014). Plant viral vectors for delivery by agrobacterium. Curr. Top. Microbiol. Immunol. 375, 155-192. doi: 10.1007/82_2013_352

Gülck, T., and Møller, B. L. (2020). Phytocannabinoids: origins and biosynthesis. Trends Plant Sci. 25, 985-1004. doi: 10.1016/j.tplants.2020.05.005

Hahn, F., Eisenhut, M., Mantegazza, O., and Weber, A. P. M. (2018). Homologydirected repair of a defective glabrous gene in arabidopsis with cas9-based gene targeting. Front. Plant Sci. 9:424. doi: 10.3389/fpls.2018.00424

Hartsel, S. C., Loh, W. H. T., and Robertson, L. W. (1983). Biotransformation of cannabidiol to Cannabielsoin by suspension cultures of Cannabis sativa and Saccharum officinarum. Planta Med. 48, 17-19. doi: 10.1055/s-2007-969870

Heitrich, A., and Binder, M. (1982). Identification of (3R, 4R)-delta 1(6)tetrahydrocannabinol as an isolation artefact of cannabinoid acids formed by callus cultures of Cannabis sativa L. Experientia 38, 898-899. doi: 10.1007/ BF01953640

Hemphill, J. K., Turner, J. C., and Mahlberg, P. G. (1978). Studies on growth and cannabinoid composition of callus derived from different strains of Cannabis sativa. Lloydia 41, 453-462.

Henry, I. M., Nagalakshmi, U., Lieberman, M. C., Ngo, K. J., Krasileva, K. V., Vasquez-Gross, H., et al. (2014). Efficient genome-wide detection and cataloging of EMS-induced mutations using exome capture and next-generation sequencing. Plant Cell 26, 1382-1397. doi: 10.1105/tpc.113.121590

Hill, A. J., Williams, C. M., Whalley, B. J., and Stephens, G. J. (2012). Phytocannabinoids as novel therapeutic agents in CNS disorders. Pharmacol. Ther. 133, 79-97. doi: 10.1016/j.pharmthera.2011.09.002

Hoerster, G., Wang, N., Ryan, L., Wu, E., Anand, A., McBride, K., et al. (2020). Use of non-integrating Zm-Wus2 vectors to enhance maize transformation. In Vitro Cell. Dev. Biol. Plant 56, 265-279. doi: 10.1007/s11627-019-10042-2

Holme, I. B., Wendt, T., and Holm, P. B. (2013). Intragenesis and cisgenesis as alternatives to transgenic crop development. Plant Biotechnol. J. 11, 395-407. doi: 10.1111/pbi.12055

Horstman, A., Li, M., Heidmann, I., Weemen, M., Chen, B., Muino, J. M., et al. (2017). The BABY BOOM transcription factor activates the LEC1-
ABI3-FUS3-LEC2 network to induce somatic embryogenesis. Plant Physiol. 175, 848-857. doi: 10.1104/pp.17.00232

Hou, H., Atlihan, N., and Lu, Z. X. (2014). New biotechnology enhances the application of cisgenesis in plant breeding. Front. Plant Sci. 5:389. doi: 10.3389/fpls.2014.00389

Husain, R., Weede, H., Bogush, D., Deguchi, M., Soliman, M., Potlakayala, S., et al. (2019). Enhanced tolerance of industrial hemp (Cannabis sativa L.) plants on abandoned mine land soil leads to overexpression of cannabinoids. PLoS One 14:e0221570. doi: 10.1371/journal.pone.0221570

Itokawa, H., Takeya, K., and Akasu, M. (1975). Studies on the constituents isolated from the callus of Cannabis sativa L. Shoyakugaku Zasshi 29, 106-112.

Izzo, A. A., Borrelli, F., Capasso, R., Di Marzo, V., and Mechoulam, R. (2009). Non-psychotropic plant cannabinoids: new therapeutic opportunities from an ancient herb. Trends Pharmacol. Sci. 30, 515-527. doi: 10.1016/j. tips.2009.07.006

Jacobs, T. B., Zhang, N., Patel, D., and Martin, G. B. (2017). Generation of a collection of mutant tomato lines using pooled CRISPR libraries. Plant Physiol. 174, 2023-2037. doi: 10.1104/pp.17.00489

Jaganathan, D., Ramasamy, K., Sellamuthu, G., Jayabalan, S., and Venkataraman, G. (2018). CRISPR for crop improvement: an update review. Front. Plant Sci. 9:985. doi: 10.3389/fpls.2018.00985

Jiang, Y., Xia, Z., Tang, Y., Han, Q., and Han, C. (2015). Preliminary studies on the tissue culture of Cannabis sativa L. (industrial hemp). Agric. Sci. Technol. 16, 923-925. doi: 10.4236/as.2018.910088

Joga, M. R., Zotti, M. J., Smagghe, G., and Christiaens, O. (2016). RNAi efficiency, systemic properties, and novel delivery methods for pest insect control: what we know so far. Front. Physiol. 7:553. doi: 10.3389/ fphys.2016.00553

Kanehisa, M., and Goto, S. (2000). KEGG: Kyoto encyclopedia of genes and genomes. Nucleic Acids Res. 28, 27-30. doi: 10.1093/nar/28.1.27

Kang, B. -C., Yun, J. -Y., Kim, S. -T., Shin, Y., Ryu, J., Choi, M., et al. (2018). Precision genome engineering through adenine base editing in plants. Nat. Plants 4, 427-431. doi: 10.1038/s41477-018-0178-x

King, J. L., Finer, J. J., and McHale, L. K. (2015). Development and optimization of agroinfiltration for soybean. Plant Cell Rep. 34, 133-140. doi: 10.1007/ s00299-014-1694-4

Koch, A., Biedenkopf, D., Furch, A., Weber, L., Rossbach, O., Abdellatef, E., et al. (2016). An RNAi-based control of Fusarium graminearum infections through spraying of long dsRNAs involves a plant passage and is controlled by the fungal silencing machinery. PLoS Pathog. 12:e1005901. doi: 10.1371/ journal.ppat.1005901

Koch, A., and Kogel, K. H. (2014). New wind in the sails: improving the agronomic value of crop plants through RNAi-mediated gene silencing. Plant Biotechnol. J. 12, 821-831. doi: 10.1111/pbi.12226

Kodym, A., and Leeb, C. J. (2019). Back to the roots: protocol for the photoautotrophic micropropagation of medicinal Cannabis. Plant Cell Tissue Organ Cult. 138, 399-402. doi: 10.1007/s11240-019-01635-1

$\mathrm{Ku}$, J., Chen, A., and Lan, E. (2020). Metabolic engineering design strategies for increasing acetyl-CoA flux. Metabolites 10:166. doi: 10.3390/metabo10040166

Lai, H., and Chen, Q. (2012). Bioprocessing of plant-derived virus-like particles of Norwalk virus capsid protein under current Good Manufacture Practice regulations. Plant Cell Rep. 31, 573-584. doi: 10.1007/s00299-011-1196-6

Lata, H., Chandra, S., Khan, I., and ElSohly, M. (2009a). Thidiazuron-induced high-frequency direct shoot organogenesis of Cannabis sativa L. In Vitro Cell Dev. Biol. Plant 45, 12-19. doi: 10.1007/s11627-008-9167-5

Lata, H., Chandra, S., Khan, I., and ElSohly, M. (2009b). Propagation through alginate encapsulation of axillary buds of Cannabis sativa L. - an important medicinal plant. Physiol. Mol. Biol. Plants 15, 79-86. doi: 10.1007/ s12298-009-0008-8

Lata, H., Chandra, S., Khan, I. A., and Elsohly, M. A. (2010). High frequency plant regeneration from leaf derived callus of high $\Delta 9$-tetrahydrocannabinol yielding Cannabis sativa L. Planta Med. 76, 1629-1633. doi: 10.1055/s-0030-1249773

Lata, H., Chandra, S., Mehmedic, Z., Khan, I., and ElSohly, M. (2012). In vitro germplasm conservation of high $\Delta^{9}$-tetrahydrocannabinol yielding elite clones of Cannabis sativa $\mathrm{L}$ under slow growth conditions. Acta. Physiol. Plant. 34, 743-750. doi: 10.1007/s11738-011-0874-x

Lata, H., Chandra, S., Khan, I., and ElSohly, M. (2016b). "In vitro propagation of Cannabis sativa L. and evaluation of regenerated plants for genetic fidelity 
and cannabinoids content for quality assurance" in Methods in molecular biology protocols for in vitro cultures and secondary metabolite analysis of aromatic and medicinal plants. 2nd Edn. ed. J. S. Mohan (New York, NY: Humana Press), 275-288.

Lata, H., Chandra, S., Khan, I. A., and Elsohly, M. A. (2017). "Micropropagation of Cannabis sativa L.-an update" in Cannabis sativa L.-Botany and biotechnology. eds. S. Chandra, H. Lata, and M. A. ElSohly (Cham: Springer International Publishing), 285-297.

Lata, H., Chandra, S., Techen, N., Khan, I. A., and ElSohly, M. A. (2016a). In vitro mass propagation of Cannabis sativa L.: a protocol refinement using novel aromatic cytokinin meta-topolin and the assessment of ecophysiological, biochemical and genetic fidelity of micropropagated plants. J. Appl. Res. Med. Aromat. Plants 3, 18-26. doi: 10.1016/j.jarmap.2015.12.001

Liu, C. C., Chi, C., Jin, L. -J., Zhu, J., Yu, J. -Q., and Zhou, Y. -H. (2018). The bZip transcription factor HY5 mediates CRY1a-induced anthocyanin biosynthesis in tomato. Plant Cell Environ. 41, 1762-1775. doi: 10.1111/ pce. 13171

Loh, W. H. T., Hartsel, S. C., and Robertson, L. W. (1983). Tissue culture of Cannabis sativa $\mathrm{L}$. and in vitro biotransformation of phenolics. $Z$. Pflanzenphysiol. 111, 395-400. doi: 10.1016/S0044-328X(83)80003-8

Lowe, K., Emily Wu, E., Wang, N., Hoerster, G., Hastings, C., Cho, M. -J., et al. (2016). Morphogenic regulators baby boom and Wuschel improve monocot transformation. J. Plant Cell 28, 1998-2015. doi: 10.1105/tpc.16.00124

Luo, J., Butelli, E., Hill, L., Parr, A., Niggeweg, R., Bailey, P., et al. (2008). AtMYB12 regulates caffeoyl quinic acid and flavonol synthesis in tomato: expression in fruit results in very high levels of both types of polyphenol. Plant J. 56, 316-326. doi: 10.1111/j.1365-313X.2008.03597.x

Luo, X., Reiter, M. A., d'Espaux, L., Wong, J., Denby, C. M., Lechner, A., et al. (2019). Complete biosynthesis of cannabinoids and their unnatural analogues in yeast. Nature 567, 123-126. doi: 10.1038/s41586-019-0978-9

Mackinnon, L., Mcdougall, G., Aziz, N., and Millam, S. (2000). "Progress towards transformation of fibre hemp." in Scottish crop research institute annual report. (Invergowrie, Dundee: Scottish Crop Research Institute), 84-86.

Maher, M., Nasti, R. A., Vollbrecht, M., Starker, C., Clark, M. D., and Voytas, D. F. (2020). Plant gene editing through de novo induction of meristems. Nat. Biotechnol. 38, 84-89. doi: 10.1038/s41587-019-0337-2

Mamta, B., and Rajam, M. V. (2017). RNAi technology: a new platform for crop pest control. Physiol. Mol. Biol. Plants 23, 487-501. doi: 10.1007/ s12298-017-0443-x

Mandolino, G., and Ranalli, P. (1999). "Advances in biotechnological approaches for hemp breeding and industry" in Advances in hemp research. ed. P. Ranalli (New York: Haworth Press), 185-208.

Marks, M. D., Tian, L., Wenger, J., Omburo, S. N., Soto-Fuentes, W., He, J., et al. (2009). Identification of candidate genes affecting Delta9tetrahydrocannabinol biosynthesis in Cannabis sativa. J. Exp. Bot. 60, 3715-3726. doi: $10.1093 / \mathrm{jxb} / \mathrm{erp} 210$

McPartland, J. M., and Russo, E. B. (2001). Cannabis and Cannabis extracts. J. Cannabis Ther. 1, 103-132. doi: 10.1300/J175v01n03_08

Méndez-Hernández, H. A., Ledezman-Rodriguez, M., Avilez-Montalvo, R. N., Juarez-Gomez, Y. L., Skeete, A. O., Avilez, J., et al. (2019). Signaling overview of plant somatic embryogenesis. Front. Plant Sci. 10:77. doi: 10.3389/ fpls.2019.00077

Mishra, R., Joshi, R. K., and Zhao, K. (2020). Base editing in crops: current advances, limitations and future implications. Plant Biotechnol. J. 18, 20-31. doi: $10.1111 /$ pbi. 13225

Mitter, N., Worrall, E. A., Robinson, K. E., Li, P., Jain, R. G., Taochy, C., et al. (2017). Clay nanosheets for topical delivery of RNAi for sustained protection against plant viruses. Nat. Plants 3:16207. doi: 10.1038/nplants.2016.207

Monsur, M. B., Shao, G., Lv, Y., Ahmad, S., Wei, X., Hu, P., et al. (2020). Base editing: the ever expanding clustered regularly interspaced short palindromic repeats (CRISPR) tool kit for precise genome editing in plants. Genes 11:466. doi: 10.3390/genes11040466

Monthony, A., Kyne, S., Grainger, C., and Jones, M. (2020). Recalcitrance of Cannabis sativa to de novo regeneration, a multi-genotype replication study. doi: 10.1101/2020.06.23.167478 [Preprint].

Mookkan, M., Nelson-Vasilchik, K., Hague, J., Zhang, Z. J., and Kausch, A. P. (2017). Selectable marker independent transformation of recalcitrant maize inbred B73 and sorghum P898012 mediated by morphogenic regulators
BABY BOOM and WUSCHEL2. Plant Cell Rep. 36, 1477-1491. doi: 10.1007/ s00299-017-2169-1

Movahedi, M., Ghasemi-Omran, V. -O., and Torabi, S. (2015). The effect of different concentrations of TDZ and BA on in vitro regeneration of Iranian Cannabis (Cannabis sativa) using cotyledon and epicotyl explants. J. Plant Mol. Breed. 3, 20-27. doi: 10.22058/jpmb.2015.15371

Movahedi, M., Ghasemi-Omran, V. O., and Torabi, S. (2016a). In vitro callus induction and regeneration of medicinal plant Cannabis sativa L. Iran. J. Med. Aromat. Plants 32, 758-769.

Movahedi, M., Ghasemi-Omran, V., and Torabi, S. (2016b). Effect of explants type and plant growth regulators on in vitro callus induction and shoot regeneration of Cannabis sativa L. Iran. J. Med. Aromat. Plants 32, 83-96.

Norkunas, K., Harding, R., Dale, J., and Dugdale, B. (2018). Improving agroinfiltration-based transient gene expression in Nicotiana benthamiana. Plant Methods 14:71. doi: 10.1186/s13007-018-0343-2

O'Connor, S. E. (2015). Engineering of secondary metabolism. Annu. Rev. Genet. 49, 71-94. doi: 10.1146/annurev-genet-120213-092053

Onofri, C., de Meijer, E. P. M., and Mandolino, G. (2015). Sequence heterogeneity of cannabidiolic- and tetrahydrocannabinolic acid-synthase in Cannabis sativa L. and its relationship with chemical phenotype. Phytochemistry 116, 57-68. doi: 10.1016/j.phytochem.2015.03.006

Outchkourov, N. S., Karlova, R., Holscher, M., Schrama, X., Blilou, I., Jongedijk, E., et al. (2018). Transcription factor-mediated control of anthocyanin biosynthesis in vegetative tissues. Plant Physiol. 176, 1862-1878. doi: 10.1104/pp.17.01662

Page, S. R. G., Monthony, A. S., and Jones, A. M. P. (2020). Basal media optimization for the micropropagation and callogenesis of Cannabis sativa L. bioRxiv: 1-23. doi: 10.1101/2020.02.07.939181 [Preprint]

Pan, Q., Wang, C., Xiong, Z., Wang, H., Fu, X., Shen, Q., et al. (2019). CrERF an $\mathrm{AP}_{2} / \mathrm{ERF}$ transcription factor, positively regulates the biosynthesis of bisindole alkaloids and their precursors in Catharanthus roseus. Front. Plant Sci. 10:931. doi: 10.3389/fpls.2019.00931

Pertwee, R. G. (2008). The diverse CB1 and CB2 receptor pharmacology of three plant cannabinoids: $\Delta 9$-tetrahydrocannabinol, cannabidiol and $\Delta 9$ tetrahydrocannabivarin. Br. J. Pharmacol. 153, 199-215. doi: 10.1038/sj. bjp. 0707442

Petrick, J. S., Brower-Toland, B., Jackson, A. L., and Kier, L. D. (2013). Safety assessment of food and feed from biotechnology-derived crops employing RNA-mediated gene regulation to achieve desired traits: a scientific review. Regul. Toxicol. Pharmacol. 66, 167-176. doi: 10.1016/j. yrtph.2013.03.008

Plawuszewski, M., Lassocinski, W., and Wieglus, K. (2006). "Regeneration of polish cultivars of monoecious hemp (Cannabis sativa L.) grown in vitro" in Renewable resources and plant biotechnology. eds. R. Kozlowski, E. Gennady and F. Pudel (Hauppauge: Nova Publishers), 149-154.

Pyne, M. E., Narcross, L., and Martin, V. J. J. (2019). Engineering plant secondary metabolism in microbial systems. Plant Physiol. 179, 844-861. doi: 10.1104/ pp.18.01291

Raharjo, T. J., Eucharia, O., Chang, W. T., and Verpoorte, R. (2010). Callus induction and phytochemical characterization of Cannabis sativa cell suspension cultures. Indian J. Chem. 6, 70-74. doi: 10.22146/ijc.21776

Reed, J., and Osbourn, A. (2018). Engineering terpenoid production through transient expression in Nicotiana benthamiana. Plant Cell Rep. 37, 1431-1441. doi: 10.1007/s00299-018-2296-3

Rotherham, D., and Harbison, S. A. (2011). Differentiation of drug and nondrug Cannabis using a single nucleotide polymorphism (SNP) assay. Forensic Sci. Int. 207, 193-197. doi: 10.1016/j.forsciint.2010.10.006

Russo, E. B. (2011). Taming THC: potential Cannabis synergy and phytocannabinoid-terpenoid entourage effects. Br. J. Pharmacol. 163, 1344-1364. doi: $10.1111 / \mathrm{j} .1476-5381.2011 .01238 . x$

Russo, E. B. (2019). The case for the entourage effect and conventional breeding of clinical Cannabis: no "strain," no gain. Front. Plant Sci. 9:1969. doi: 10.3389/fpls.2018.01969

Salentijn, E. M. J., Petit, J., and Trindade, L. M. (2019). The complex interactions between flowering behavior and fiber quality in hemp. Front. Plant Sci. 10:614. doi: 10.3389/fpls.2019.00614

Sawler, J., Stout, J. M., Gardner, K. M., Hudson, D., Vidmar, J., Butler, L., et al. (2015). The genetic structure of marijuana and hemp. PLoS One 10:e0133292. doi: 10.1371/journal.pone.0133292 
Schachtsiek, J., Hussain, T., Azzouhri, K., Kayser, O., and Stehle, F. (2019). Virus-induced gene silencing (VIGS) in Cannabis sativa L. Plant Methods 15:157. doi: 10.1186/s13007-019-0542-5

Schachtsiek, J., Warzecha, H., Kayser, O., and Stehle, F. (2018). Current perspectives on biotechnological cannabinoid production in plants. Planta Med. 84, 214-220. doi: 10.1055/s-0043-125087

Schluttenhofer, C., and Yuan, L. (2017). Challenges towards revitalizing hemp: a multifaceted crop. Trends Plant Sci. 22, 917-929. doi: 10.1016/j. tplants.2017.08.004

Shen, J. P., Zhao, D., Sasik, R., Luebeck, J., Birmingham, A., Bojorquez-Gomez, A., et al. (2017). Combinatorial CRISPR-Cas9 screens for de novo mapping of genetic interactions. Nat. Methods 14, 573-576. doi: 10.1038/nmeth.4225

Shrestha, A., Khan, A., and Dey, N. (2018). Cis-trans engineering: advances and perspectives on customized transcriptional regulation in plants. Mol. Plant 11, 886-898. doi: 10.1016/j.molp.2018.05.008

Si, X., Zhang, H., Wang, Y., Chen, K., and Gao, C. (2020). Manipulating gene translation in plants by CRISPR-Cas9-mediated genome editing of upstream open reading frames. Nat. Protoc. 15, 338-363. doi: 10.1038/s41596-019-0238-3

Sirikantaramas, S., Morimoto, S., Shoyama, Y., Ishikawa, Y., Wada, Y., Shoyama, Y., et al. (2004). The gene controlling marijuana psychoactivity: molecular cloning and heterologous expression of Delta1-tetrahydrocannabinolic acid synthase from Cannabis sativa L. J. Biol. Chem. 279, 39767-39774. doi: 10.1074/jbc. M403693200

Sirikantaramas, S., Taura, F., Tanaka, Y., Ishikawa, Y., Morimoto, S., and Shoyama, Y. (2005). Tetrahydrocannabinolic acid synthase, the enzyme controlling marijuana psychoactivity, is secreted into the storage cavity of the glandular trichomes. Plant Cell Physiol. 46, 1578-1582. doi: 10.1093/ $\mathrm{pcp} / \mathrm{pci} 166$

Sirkowski, E. (2012). Marked Cannabis for indicating medical Marijuana. US Patent 20120311744.

Slusarkiewicz-Jarzina, A., Ponitka, A., and Kaczmarek, Z. (2005). Influence of cultivar, explant source and plant growth regulator on callus induction and plant regeneration of Cannabis sativa L. Acta Biol. Crac. Ser. Bot. 47, 145-151.

Smýkalová, I., Vrbová, M., Cvečková, M., and Plačková, L. (2019). The effects of novel synthetic cytokinin derivatives and endogenous cytokinins on the in vitro growth responses of hemp (Cannabis sativa L.) explants. Plant Cell Tissue Organ Cult. 139, 381-394. doi: 10.1007/s11240-019-01693-5

Stout, J. M., Boubakir, Z., Ambrose, S. J., Purves, R. W., and Page, J. E. (2012). The hexanoyl-CoA precursor for cannabinoid biosynthesis is formed by an acyl-activating enzyme in Cannabis sativa trichomes. Plant J. 71, 353-365. doi: $10.1111 /$ j.1365-313X.2012.04949.x

Stracke, R., Ishihara, H., Huep, G., Barsch, A., Mehrtens, F., Niehaus, K., et al. (2007). Differential regulation of closely related R2R3-MYB transcription factors controls flavonol accumulation in different parts of the Arabidopsis thaliana seedling. Plant J. 50, 660-677. doi: 10.1111/j.1365-313X.2007.03078.x

Sun, Y., Jiao, G., Liu, Z., Zhang, X., Li, J., Guo, X., et al. (2017). Generation of high-amylose rice through CRISPR/Cas9-mediated targeted mutagenesis of starch branching enzymes. Front. Plant Sci. 8:298. doi: 10.3389/ fpls.2017.00298

Thacker, X., Thomas, K., Fuller, M., and Smith, S. (2018). Determination of optimal hormone and mineral salts levels in tissue culture media for callus induction and growth of industrial hemp (Cannabis sativa L.). Agric. Sci. 9, 1250-1268. doi: 10.4236/as.2018.910088

Valliere, M. A., Korman, T., Woodall, N., Khitrov, G. A., Taylor, R. E., Baker, D., et al. (2019). A cell-free platform for the prenylation of natural products and application to cannabinoid production. Nat. Commun. 10:565. doi: 10.1038/s41467-019-08448-y

van Bakel, H., Stout, J. M., Cote, A. G., Tallon, C. M., Sharpe, A. G., Hughes, T. R., et al. (2011). The draft genome and transcriptome of Cannabis sativa. Genome Biol. 12:R102. doi: 10.1186/gb-2011-12-10-r102
Van Moerkercke, A., Steensma, P., Schweizer, F., Pollier, J., Gariboldi, I., Payne, R., et al. (2015). The bHLH transcription factor BIS1 controls the iridoid branch of the monoterpenoid indole alkaloid pathway in Catharanthus roseus. Proc. Natl. Acad. Sci. U. S. A. 112, 8130-8135. doi: 10.1073/pnas.1504951112

Veliky, I. A., and Genest, K. (1972). Growth and metabolites of Cannabis sativa cell suspension cultures. Lloydia 35, 450-456.

Vincent, D., Binos, S., Rochfort, S., and Spangenberg, G. (2019). Top-down proteomics of medicinal Cannabis. Proteomes 7:33. doi: 10.3390/proteomes7040033

Wahby, I., Caba, J. M., and Ligero, F. (2013). Agrobacterium infection of hemp (Cannabis sativa L.): establishment of hairy root cultures. J. Plant Interact. 8, 312-320. doi: 10.1080/17429145.2012.746399

Wahby, I., Caba, J. M., and Ligero, F. (2017). "Hairy root culture as a biotechnological tool in C. sativa" in Cannabis sativa L. - Botany and biotechnology. eds. S. Chandra, H. Lata, and M. A. ElSohly (Berlin: Springer), 299-317.

Wang, R., He, L. S., Xia, B., Tong, J. F., Li, N., and Peng, F. (2009). A micropropagation system for cloning of hemp (Cannabis sativa L.) by shoot tip culture. Pak. J. Bot. 41, 603-608.

Wang, Y., Zhang, H., Li, H., and Miao, X. (2011). Second-generation sequencing supply an effective way to screen RNAi targets in large scale for potential application in pest insect control. PLoS One 6:e18644. doi: 10.1371/journal. pone. 0018644

Weiblen, G. D., Wenger, J. P., Craft, K. J., ElSohly, M. A., Mehmedic, Z., Treiber, E. L., et al. (2015). Gene duplication and divergence affecting drug content in Cannabis sativa. New Phytol. 208, 1241-1250. doi: 10.1111/ nph.13562

Wielgus, K., Luwanska, A., Lassocinski, W., and Kaczmarek, Z. (2008). Estimation of Cannabis sativa L. tissue culture conditions essential for callus induction and plant regeneration. J. Nat. Fibers 5, 199-207. doi: 10.1080/ 15440470801976045

Wolter, F., Schindele, P., and Puchta, H. (2019). Plant breeding at the speed of light: the power of CRISPR/Cas to generate directed genetic diversity at multiple sites. BMC Plant Biol. 19:176. doi: 10.1186/s12870-019-1775-1

Wróbel, T., Dreger, M., Wielgus, K., and Słomski, R. (2020). Modified nodal cuttings and shoot tips protocol for rapid regeneration of Cannabis sativa L. J. Nat. Fibers 19, 1-10. doi: 10.1080/15440478.2020.1748160

Zager, J. J., Lange, I., Srividya, N., Smith, A., and Lange, B. M. (2019). Gene networks underlying cannabinoid and terpenoid accumulation in Cannabis. Plant Physiol. 180, 1877-1897. doi: 10.1104/pp.18.01506

Zhang, W. J., and Björn, L. O. (2009). The effect of ultraviolet radiation on the accumulation of medicinal compounds in plants. Fitoterapia 80, 207-218. doi: $10.1016 /$ j.fitote.2009.02.006

Zirpel, B., Degenhardt, F., Martin, C., Kayser, O., and Stehle, F. (2017). Engineering yeasts as platform organisms for cannabinoid biosynthesis. J. Biotechnol. 259, 204-212. doi: 10.1016/j.jbiotec.2017.07.008

Zuardi, A. W. (2008). Cannabidiol: from an inactive cannabinoid to a drug with wide spectrum of action. Br. J. Psychiatry 30, 271-280. doi: 10.1590/ s1516-44462008000300015

Conflict of Interest: The authors declare that the research was conducted in the absence of any commercial or financial relationships that could be construed as a potential conflict of interest.

Copyright (c) 2020 Deguchi, Kane, Potlakayala, George, Proano, Sheri, Curtis and Rudrabhatla. This is an open-access article distributed under the terms of the Creative Commons Attribution License (CC BY). The use, distribution or reproduction in other forums is permitted, provided the original author(s) and the copyright owner(s) are credited and that the original publication in this journal is cited, in accordance with accepted academic practice. No use, distribution or reproduction is permitted which does not comply with these terms. 